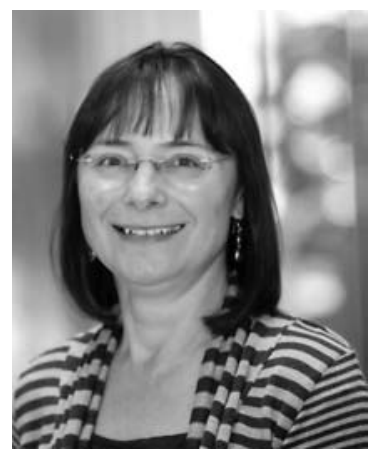

\title{
A Conversation with Karen Vousden
}

\author{
INTERVIEWER: JAN WITKOWSKI \\ Executive Director, Banbury Center at Cold Spring Harbor Laboratory
}

Karen Vousden is Director of the Cancer Research UK Beatson Institute.

Jan Witkowski: Over the past few years, there's been a markedly increasing emphasis and interest in the metabolism and biochemistry of cancers. It's moving away from just the genes and proteins. Am I right in thinking that?

Dr. Vousden: Yes, I think you're absolutely right. There's been a resurgence of interest in metabolism. In the middle of the last century a lot of energy was spent on understanding metabolism and biochemists at this time were making huge progress. Many metabolic pathways were worked out in exquisite detail in the '50s and the '60s. Then came the oncogene revolution, and much of the emphasis switched to understanding the regulation of the cell cycle and signal transduction. At this point, metabolism was almost forgotten in cancer biology until about 10 years, when there was a reemergence of interest. Craig Thompson was showing a big poster of all the cancer-signaling pathways, and another with all the metabolic pathways, pointing out that there was not a single gene on one that's also in the other. I think we are fixing that now.

Jan Witkowski: Was there any particular event or finding that has led to this increased interest in biochemistry?

Dr. Vousden: I have to attribute a lot of this to the work of a few labs who led the way in this reemergence of tumor metabolism. This was provoked by the general realization that control of the cell cycle is not enough to explain cancer biology, and that cells need to grow as well as divide. As our understanding of the regulation of the cell cycle grew, the community started to look beyond cell division to the importance of other aspect of proliferation.

Jan Witkowski: Warburg came up with the idea that aerobic glycolysis was a characteristic feature of cancer cells. In modern findings, has that proved true at all?

Dr. Vousden: It has held up. In the end, Warburg's observation is what underlies PET (positron emission tomography) imaging. It certainly is the case that many cancers have a high rate of glucose uptake, which is what we measure when we do functional imaging, or a PET scan.

However, over recent years our understanding of the Warburg effect has become more sophisticated, although we still don't quite understand it. We know now that there probably are changes in the metabolism of almost all cancers. We're interested in understanding that and then asking, "Can we harness any of this information to develop new therapies?"

Jan Witkowski: Can you tell me a little about your current work?

Dr. Vousden: We became very interested in a specific aspect of metabolism, which is how cells use and produce the amino acid serine and why changes in serine metabolism might be particularly important in cancers. Although serine is not an essential amino acid - cells can make their own serine - tumors can be broadly divided into two groups. One group has amplifications in the de novo serine synthesis pathway, so these tumors can make a lot of serine from glucose. The others seem to depend more heavily on exogenous serine, although many of these cancers can switch to making their own serine when the external supply is taken away. This adaptation to serine starvation is a point of vulnerability for those cells, which we are hoping to exploit to deliver a therapeutic advantage.

Jan Witkowski: Why is serine such an important amino acid?

Dr. Vousden: Obviously it's important for the synthesis of proteins and it can also be used to make other amino acids. But it is also a major one-carbon donor to support the folate cycle. The folate cycle is necessary for nucleotide synthesis - purine and thymidine synthesis - and also contributes to the SAM ( $S$-adenosyl methionine) cycle, and all the methylation reactions that we are hearing

(C) 2016 Vousden. This article is distributed under the terms of the Creative Commons Attribution-NonCommercial License, which permits reuse and redistribution, except for commercial purposes, provided that the original author and source are credited. 
about. Interestingly, some of the first successful chemotherapeutics work by targeting the folate cycle. We are now exploring additional functions of one-carbon metabolism that might be playing a role in providing ATP, $\mathrm{NADH}$, and NADPH.

Jan Witkowski: Did you start with just serine? Did you just come across it all or was this a carefully thought-out step?

Dr. Vousden: Oh, it was definitely not a carefully thought-out step! My primary interest has been with the investigation of how the tumor suppressor p53 works. We've been very interested in the past in understanding how p53 arrests and kills cells. I can't even remember how this came up, but we did begin to realize that having p53 is not always detrimental to the survival of the cell and that under some circumstances p53 actually helps cells cope with certain types of stress. Those stresses tended to be mild metabolic, nongenotoxic stresses, stresses that you can easily imagine being reversible.

Previous work had shown that cells with p53 deal better with low levels of glucose than cells lacking p53. That was a really interesting observation, because it was paradoxical to the general canonical activities of p53, which are to stop cells from growing by arresting or killing them. We thought, "What about other types of starvation?" Oliver Maddocks, a postdoc in the lab, simply tested the requirement for every amino acid and found that serine starvation made cancer cells grow less well, but that growth was further reduced if they lost p53. That was our starting point. At the same time, several other groups-Matt Vander Heiden, David Sabatini, Lew Cantley-all made observations that showed serine synthesis was going to be important in cancers. It was really great because we were all working on the same problem that I think no one had really considered 5 years previously.

Jan Witkowski: Are you working on enzymes in the serine synthesis pathway?

Dr. Vousden: There are several aspects of this. We're still very interested in the idea that p53 can provide survival functions under the conditions of nutrient stress. We see this not only with serine, but also with glutamine and we are looking at other types of nutrients now. Another interesting thought is around mutant $\mathrm{p} 53$. We have found that while cells that don't have any p53 are more vulnerable to this type of stress, some of the p53 mutations that occur in cancer support survival similarly to wild-type p53. This seems to make sense-these tumor-derived mutants have lost the "death" activities of p53 but they retain survival functions. Interestingly, these are the mutations that are associated with the worst prognosis.

In terms of serine synthesis and the one-carbon cycle, we are looking at drugs that target enzymes in serine synthesis pathways, as well as some of the downstream enzymes that are involved in the metabolism of serine. We have been interested in a step that produces mitochondrial NADPH, which may be important for antioxidant activities. But our particular take on this whole area has been to simply say, "Can we see a therapeutic effect by lowering circulating serine levels simply by taking serine out of the diet?"

Jan Witkowski: How easy is it to affect serine levels like that?

Dr. Vousden: We can give mice a completely serinedeficient diet, and we have been using various different iterations of this with diets lacking various combinations of amino acids. Interestingly, and much to my surprise,Oliver carried on doing this even though I said it wouldn't ever work - you can reduce serum serine levels by $\sim 50 \%$ simply by giving mice serine-free diets.

There are examples where in-built errors of metabolism can be rescued by modulating amino acids in the diet in humans. Treatment of phenylketonuria by limiting phenylalanine is the classic example. Then there are syndromes leading to the accumulation of glycine, where treatments to reduce serum glycine levels are used to try to rescue some neurological phenotypes, as well as defects in serine synthesis pathway enzymes that can be rescued by increasing dietary serine. So it's certainly possible, and we are talking to some companies now to see whether we can try to at least formulate a diet to determine whether we can lower serine levels in people.

Jan Witkowski: How much serine is in the typical diet?

Dr. Vousden: Protein-rich foods like meat, cheese, and eggs are all high in serine, and most people won't be serine-deprived. I think that it would be difficult to really limit serine without having an absolutely synthetic diet, which would be, of course, very problematic to administer.

We have clues from our animal models that there is synergy between serine limitation and ROS (reactive oxygen species) activation, leading to an enhanced vulnerability that means cells adapting to serine starvation are more sensitive to increases in oxidative stress. Fortunately, many common chemotherapies induce ROS. With our clinical colleagues we are now considering whether we could carry out a clinical trial where we move patients onto a serine-free diet only over the course of their chemotherapy. In this way they wouldn't go on this diet forever, but we may be able to increase the efficacy of the chemotherapy by using the diet for only a week or two.

Jan Witkowski: That makes a great deal of sense because then it's not such a burden on the patient. I presume these diets are not going to be inexpensive.

Dr. Vousden: There are companies who make these sorts of diets for clinical use, and simply reformulating them to take out serine rather than some of the other amino acids would be relatively straightforward. I don't know what the expense in the end would be, but I think it depends on how effective it becomes.

The other important consideration at the moment is whether there will be any side effects that aren't associated with the tumor. The most common concern I hear is 
neurological defects. Obviously, serine and glycine are very important in the brain. That, I think, is more of a problem for the inhibition of serine synthesis pathways because the brain is where cells make their own serine. So we are probably less likely to have a neurological effect in response to removing serine from the diet.

Jan Witkowski: Do cells generally prefer to make their own serine?

Dr. Vousden: Most tumor cells, we think, prefer to take it up, and that's why we can have an effect by taking it away from the circulation. But the response is very varied. We are working out now how the genetic constitution of the tumor affects their sensitivity to exogenous serine. Obviously, tumors that have already ramped up serine synthesis won't care, but most of the other cancers are sensitive to some degree, and we would like to understand this more fully, so we can target those cancers that depend on exogenous serine the most.

Jan Witkowski: Are there particular cancers that ramp up their synthesis of serine?

Dr. Vousden: This had been shown in breast cancers, melanoma, and other tumor types. The initial observations were that $10 \%-20 \%$ of breast cancers show evidence of amplification of one of the three serine synthesis pathway enzymes, although this is higher in ER-negative tumors. It's all very interesting because these tumors depend on the serine synthesis pathway, even when they have access to exogenous serine. We're still trying to understand why that might be. 


\section{$\$_{\text {CSH\& }}^{\infty}$ Cold Spring Harbor Symposia SYMPOSIA}

\section{A Conversation with Karen Vousden}

Cold Spring Harb Symp Quant Biol 2016 81: 351-353 originally published online March 9, 2017 Access the most recent version at doi:10.1101/sqb.2016.81.031930

Creative This article is distributed under the terms of the

Commons http://creativecommons.org/licenses/by-nc/4.0/, which permits reuse and

License redistribution, except for commercial purposes, provided that the original author and source are credited.

Email Alerting Receive free email alerts when new articles cite this article - sign up in Service the box at the top right corner of the article or click here. 